\title{
Superconductivity and Superfluidity-Part II: Superconductivity as a Consequence of Ordering of Zero-point Oscillations in Electron Gas
}

\author{
Boris V.Vasiliev \\ *Corresponding Author: bv.vasiliev@narod.ru
}

Copyright (C)2014 Horizon Research Publishing All rights reserved.

\begin{abstract}
Currently there is a common belief that the explanation of superconductivity phenomenon lies in understanding the mechanism of the formation of electron pairs. Paired electrons, however, cannot form a superconducting condensate spontaneously. These paired electrons perform disorderly zero-point oscillations and there are no force of attraction in their ensemble. In order to create a unified ensemble of particles, the pairs must order their zero-point fluctuations so that an attraction between the particles appears. As a result of this ordering of zero-point oscillations in the electron gas, superconductivity arises. This model of condensation of zero-point oscillations creates the possibility of being able to obtain estimates for the critical parameters of elementary superconductors, which are in satisfactory agreement with the measured data. On the another hand, the phenomenon of superfluidity in He- 4 and $\mathrm{He}-3$ can be similarly explained, due to the ordering of zero-point fluctuations. It is therefore established that both related phenomena are based on the same physical mechanism.
\end{abstract}

Keywords Superconductivity Superfluidity Zeropoint Oscillations

\section{Superconductivity as a conse- quence of ordering of zero- point oscillations}

Superfluidity and superconductivity, which can be regarded as the superfluidity of the electron gas, are related phenomena. The main feature of these phenomena can be seen in a fact that a special condensate in superconductors as well as in superfluid helium is formed from particles interconnected by attraction.

This mutual attraction does not allow a scattering of individual particles on defects and walls, if the energy of this scattering is less than the energy of attraction. Due to the lack of scattering, the condensate acquires ability to move without friction.

Superconductivity was discovered over a century ago, and the superfluidity about thirty years later.

However, despite the attention of many scientists to the study of these phenomena, they have been the great mysteries in condensed matter physics for a long time. This mystery attracted the best minds of the twentieth century.

The mystery of the superconductivity phenomenon has begun to drop in the middle of the last century when the effect of magnetic flux quantization in superconducting cylinders was discovered and investigated. This phenomenon was predicted even before the WWII by brothers F. London and H. London, but its quantitative study were performed only two decades later.

By these measurements it became clear that at the formation of the superconducting state, two free electrons are combined into a single boson with zero spin and zero momentum.

Around the same time, it was observed that the substitution of one isotope of the superconducting element to another leads to a changing of the critical temperature of superconductors: the phenomenon called an isotopeeffect [1], [2]. This effect was interpreted as the direct proof of the key role of phonons in the formation of the superconducting state.

Following these understandings,

L. Cooper proposed the phonon mechanism of electron pairing on which base the microscopic theory of superconductivity (so called BCS-theory) was built by N. Bogolyubov and J. Bardeen, L. Cooper and J. Shrieffer (probably it should be named better the BogolyubovBCS-theory).

However the B-BCS theory based on the phonon mechanism brokes a hypothetic link between superconductivity and superfluidity as in liquid helium there are no phonons for combining atoms.

Something similar happened with the description of superfluidity.

Soon after discovery of superfluidity, L.D. Landau in his first papers on the subject immediately demonstrated that the superfluidity should be considered as a result of condensate formation consisting of macroscopic number of atoms in the same quantum state and obeying quantum laws. It gave the possibility to describe the main features of this phenomenon: the temperature de- 
pendence of the superfluid phase density, the existence of the second sound, etc. But it does not gave an answer to the question which physical mechanism leads to the unification of the atoms in the superfluid condensate and what is the critical temperature of the condensate, i.e. why the ratio of the temperature of transition to the superfluid state to the boiling point of helium- 4 is almost exactly equals to $1 / 2$.

On the whole, the description of both superphenomena, superconductivity and superfluidity, to the beginning of the

twenty first century induced some feeling of dissatisfaction primarily due to the fact that a common mechanism of their occurrence has not been understood.

More than fifty years of a study of the B-BCS-theory has shown that this theory successfully describes the general features of the phenomenon, but it can not be developed in the theory of superconductors. It explains general laws such as the emergence of the energy gap, the behavior of specific heat capacity, the flux quantization, etc., but it can not predict the main parameters of the individual superconductors: their critical temperatures and critical magnetic fields. More precisely, in the BBCS-theory, the expression for the critical temperature of superconductor obtains an exponential form which exponential factor is impossible to measure directly and this formula is of no practical interest.

Recent studies of the isotopic substitution showed that zero-point oscillations of the ions in the metal lattice are not harmonic. Consequently the isotopic substitution affects the interatomic distances in a lattice, and as the result, they directly change the Fermi energy of a metal [3].

Therefore, the assumption developed in the middle of the last century, that the electron-phonon interaction is the only possible mechanism of superconductivity was proved to be wrong. The direct effect of isotopic substitution on the Fermi energy gives a possibility to consider the superconductivity without the phonon mechanism.

Furthermore, a closer look at the problem reveals that the B-BCS-theory describes the mechanism of electron pairing, but in this theory there is no mechanism for combining pairs in the single super-ensemble. The necessary condition for the existence of superconductivity is formation of a unique ensemble of particles. By this mechanism, a very small amount of electrons are combined in super-ensemble, on the level 10 in minus fifth power from the full number of free electrons. This fact also can not be understood in the framework of the BBCS theory.

An operation of the mechanism of electron pairing and turning them into boson pairs is a necessary but not sufficient condition for the existence of a superconducting state. Obtained pairs are not identical at any such mechanism. They differ because of their uncorrelated zero-point oscillations and they can not form the condensate at that.

At very low temperatures, that allow superfluidity in helium and superconductivity in metals, all movements of particles are freezed except for their zero-point oscillations. Therefore, as an alternative, we should consider the interaction of super-particles through electromagnetic fields of zero-point oscillations. This approach was proved to be fruitful. At the consideration of superphenomena as consequences of the zero-point oscillations ordering, one can construct theoretical mechanisms enabling to give estimations for the critical parameters of these phenomena which are in satisfactory agreement with measurements.

As result, one can see that as the critical temperatures of (type-I) superconductors are equal to about $10^{-6}$ from the Fermi temperature for superconducting metal, which is consistent with data of measurements. At this the destruction of superconductivity by application of critical magnetic field occurs when the field destroys the coherence of zero-point oscillations of electron pairs. This is in good agreement with measurements also.

A such-like mechanism works in superfluid liquid helium. The problem of the interaction of zero-point oscillations of the electronic shells of neutral atoms in the s-state, was considered yet before the World War II by F.London. He has shown that this interaction is responsible for the liquefaction of helium. The closer analysis of interactions of zero-point oscillations for helium atomic shells shows that at first at the temperature of about $4 \mathrm{~K}$ only, one of the oscillations mode becomes ordered. As a result, the forces of attraction appear between atoms which are need for helium liquefaction. To create a single quantum ensemble, it is necessary to reach the complete ordering of atomic oscillations. At the complete ordering of oscillations at about $2 \mathrm{~K}$, the additional energy of the mutual attraction appears and the system of helium- 4 atoms transits in superfluid state. To form the superfluid quantum ensemble in Helium-3, not only the zero-point oscillations should be ordered, but the magnetic moments of the nuclei should be ordered too. For this reason, it is necessary to lower the temperature below $0.001 \mathrm{~K}$. This is also in agreement with experiment.

Thus it is possible to show that both related superphenomena, superconductivity and superfluidity, are based on the single physical mechanism: the ordering of zero-point oscillations.

The roles of zero-point oscillations in formation of the superconducting state have been previously considered in papers [4]-[6].

\section{The electron pairing}

J.Bardeen was first who turned his attention toward a possible link between superconductivity and zero-point oscillations [7].

The special role of zero-point vibrations exists due to the fact that at low temperatures all movements of electrons in metals have been frozen except for these oscillations.

Superconducting condensate formation requires two mechanisms: first, the electrons must be united in boson pairs, and then the zero-point fluctuations must be ordered (see Fig.(1)).

The energetically favorable pairing of electrons in the electron gas should occur above the critical temperature.

Possibly, the pairing of electrons can occur due to the magnetic dipole-dipole interaction.

For the magnetic dipole-dipole interaction, to merge two electrons into the singlet pair at the temperature of 


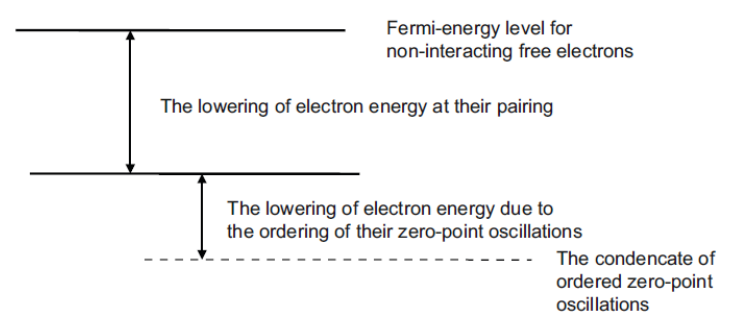

Figure 1. The schematic representation of the energy levels of conducting electrons in a superconducting metal

about $10 \mathrm{~K}$, the distance between these particles must be small enough:

$$
r<\left(\mu_{B}^{2} / k T_{c}\right)^{1 / 3} \approx a_{B}
$$

where $a_{B}=\frac{\hbar^{2}}{m_{e} e^{2}}$ is the Bohr radius.

That is, two collectivized electrons must be localized in one lattice site volume. It is agreed that the superconductivity can occur only in metals with two collectivized electrons per atom, and cannot exist in the monovalent alkali and noble metals.

It is easy to see that the presence of magnetic moments on ion sites should interfere with the magnetic combination of electrons. This is confirmed by the experimental fact: as there are no strong magnetic substances among superconductors, so adding of iron, for example, to traditional superconducting alloys always leads to a lower critical temperature.

On the other hand, this magnetic coupling should not be destroyed at the critical temperature. The energy of interaction between two electrons, located near one lattice site, can be much greater. This is confirmed by experiments showing that throughout the period of the magnetic flux quantization, there is no change at the transition through the critical temperature of superconductor [8], [9].

The outcomes of these experiments are evidence that the existence of the mechanism of electron pairing is a necessary but not a sufficient condition for the existence of superconductivity.

The magnetic mechanism of electronic pairing proposed above can be seen as an assumption which is consistent with the measurement data and therefore needs a more detailed theoretic consideration and further refinement.

On the other hand, this issue is not very important in the grander scheme, because the nature of the mechanism that causes electron pairing is not of a significant importance. Instead, it is important that there is a mechanism which converts the electronic gas into an ensemble of charged bosons with zero spin in the considered temperature range (as well as in a some range of temperatures above $T_{c}$ ).

If the temperature is not low enough, the electronic pairs still exist but their zero-point oscillations are disordered.

Upon reaching the $T_{c}$, the interaction between zeropoint oscillations should cause their ordering and therefore a superconducting state is created.

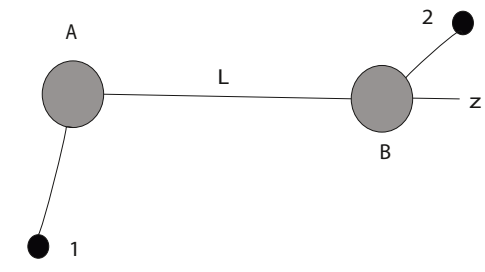

Figure 2. Two ions placed on the distance $L$ and centers of their electronic clouds.

\section{The condensate of ordered zero-point oscillations of elec- tron gas}

\subsection{The interaction of zero-point oscilla- tions}

The principal condition for the superconducting state formation is the ordering of zero-point oscillations. It is realized because the paired electrons obeying BoseEinstein statistics attract each other.

The origin of this attraction can be explained as follows.

Let two ion A and B be located on the $\mathrm{z}$ axis at the distance $\mathrm{L}$ from each other. Two collectivized electrons create clouds with centers at points 1 and 2 in the vicinity of each ions (Figure2). Let $r_{1}$ be the radius-vector of the center of the first electronic cloud relative to the ion $\mathrm{A}$ and $r_{2}$ is the radius-vector of the second electron relative to the ion $\mathrm{B}$.

Following the Born-Oppenheimer approximation, slowly oscillating ions are assumed fixed. Let the temperature be low enough $(T \rightarrow 0)$, so only zero-point fluctuations of electrons would be taken into consideration.

In this case, the Hamiltonian of the system can be written as:

$$
\begin{aligned}
& H=H_{0}+H^{\prime} \\
& \begin{aligned}
H_{0}=-\frac{\hbar^{2}}{4 m_{e}}\left(\nabla_{1}^{2}+\right. & \left.\nabla_{2}^{2}\right)- \\
& \quad-\frac{4 e^{2}}{r_{1}}-\frac{4 e^{2}}{r_{2}}
\end{aligned} \\
& H^{\prime}=\frac{4 e^{2}}{L}+\frac{4 e^{2}}{r_{12}}-\frac{4 e^{2}}{r_{1 B}}-\frac{4 e^{2}}{r_{2 A}}
\end{aligned}
$$

Eigenfunctions of the unperturbed

Hamiltonian describes two ions

surrounded by electronic clouds without interactions between them. Due to the fact that the distance between the ions is large compared with the size of the electron clouds $L \gg r$, the additional term $H^{\prime}$ characterizing the interaction can be regarded as a perturbation.

If we are interested in the leading term of the interaction energy for L, the function $H^{\prime}$ can be expanded in a series in powers of $1 / L$ and we can write the first term: 


$$
\begin{aligned}
& H^{\prime}=\frac{4 e^{2}}{L}\left\{1+\left[1+\frac{2\left(z_{2}-z_{1}\right)}{L}+\right.\right. \\
&\left.+\frac{\left(x_{2}-x_{1}\right)^{2}+\left(y_{2}-y_{1}\right)^{2}+\left(z_{2}-z_{1}\right)^{2}}{L^{2}}\right]^{-1 / 2}- \\
&-\left(1-\frac{2 z_{1}}{L}+\frac{r_{1}^{2}}{L^{2}}\right)^{-1 / 2}- \\
&\left.-\left(1+\frac{2 z_{2}}{L}+\frac{r_{2}^{2}}{L^{2}}\right)^{-1 / 2}\right\} .
\end{aligned}
$$

After combining the terms in this expression, we get:

$$
H^{\prime} \approx \frac{4 e^{2}}{L^{3}}\left(x_{1} x_{2}+y_{1} y_{2}-2 z_{1} z_{2}\right) .
$$

This expression describes the interaction of two dipoles $d_{1}$ and $d_{2}$, which are formed by fixed ions and electronic clouds of the corresponding instantaneous configuration.

Let us determine the displacements of electrons which lead to an attraction in the system .

Let zero-point fluctuations of the dipole moments formed by ions with their electronic clouds occur with the frequency $\Omega_{0}$, whereas each dipole moment can be decomposed into three orthogonal projection $d_{x}=$ $e x, d_{y}=e y$ and $d_{z}=e z$, and fluctuations of the second clouds are shifted in phase on $\varphi_{x}, \varphi_{y}$ and $\varphi_{z}$ relative to fluctuations of the first.

As can be seen from Eq.(4), the interaction of zcomponents is advantageous at in-phase zero-point oscillations of clouds, i.e., when $\varphi_{z}=2 \pi$.

Since the interaction of oscillating electric dipoles is due to the occurrence of oscillating electric field generated by them, the phase shift on $2 \pi$ means that attracting dipoles are placed along the z-axis on the wavelength $\Lambda_{0}$ :

$$
L_{z}=\Lambda_{0}=\frac{c}{2 \pi \Omega_{0}} .
$$

As follows from (4), the attraction of dipoles at the interaction of the $\mathrm{x}$ and $\mathrm{y}$-component will occur if these oscillations are in antiphase, i.e. if the dipoles are separated along these axes on the distance equals to half of the wavelength:

$$
L_{x, y}=\frac{\Lambda_{0}}{2}=\frac{c}{4 \pi \Omega_{0}}
$$

In this case

$$
H^{\prime}=-4 e^{2}\left(\frac{x_{1} x_{2}}{L_{x}^{3}}+\frac{y_{1} y_{2}}{L_{y}^{3}}+2 \frac{z_{1} z_{2}}{L_{z}^{3}}\right) .
$$

Assuming that the electronic clouds have isotropic oscillations with amplitude $a_{0}$ for each axis

$$
x_{1}=x_{2}=y_{1}=y_{2}=z_{1}=z_{2}=a_{0}
$$

we obtain

$$
H^{\prime}=576 \pi^{3} \frac{e^{2}}{c^{3}} \Omega_{0}^{3} a_{0}^{2}
$$

\subsection{The zero-point oscillations ampli- tude}

The principal condition for the superconducting state formation, that is the ordering of zero-point oscillations, is realized due to the fact that the paired electrons, which obey Bose-Einstein statistics, interact with each other.

At they interact, their amplitudes, frequencies and phases of zero-point oscillations become ordered.

Let an electron gas has density $n_{e}$ and its Fermienergy be $\mathcal{E}_{F}$. Each electron of this gas can be considered as fixed inside a cell with linear dimension $\lambda_{F}:{ }^{1}$

$$
\lambda_{F}^{3}=\frac{1}{n_{e}}
$$

which corresponds to the de Broglie wavelength:

$$
\lambda_{F}=\frac{2 \pi \hbar}{p_{F}} .
$$

Having taken into account (11), the Fermi energy of the electron gas can be written as

$$
\mathcal{E}_{F}=\frac{p_{F}^{2}}{2 m_{e}}=2 \pi^{2} \frac{e^{2} a_{B}}{\lambda_{F}^{2}} .
$$

However, a free electron interacts with the ion at its zero-point oscillations. If we consider the ions system as a positive background uniformly spread over the cells, the electron inside one cell has the potential energy:

$$
\mathcal{E}_{p} \simeq-\frac{e^{2}}{\lambda_{F}}
$$

As zero-point oscillations of the electron pair are quantized by definition, their frequency and amplitude are related

$$
m_{e} a_{0}^{2} \Omega_{0} \simeq \frac{\hbar}{2}
$$

Therefore, the kinetic energy of electron undergoing zero-point oscillations in a limited region of space, can be written as:

$$
\mathcal{E}_{k} \simeq \frac{\hbar^{2}}{2 m_{e} a_{0}^{2}}
$$

In accordance with the virial theorem [10], if a particle executes a finite motion, its potential energy $\mathcal{E}_{p}$ should be associated with its kinetic energy $\mathcal{E}_{k}$ through the simple relation $\left|\mathcal{E}_{p}\right|=2 \mathcal{E}_{k}$.

In this regard, we find that the amplitude of the zeropoint oscillations of an electron in a cell is:

$$
a_{0} \simeq \sqrt{2 \lambda_{F} a_{B}}
$$

\subsection{The condensation temperature}

Hence the interaction energy, which unites particles into the condensate of ordered zero-point oscillations

$$
\Delta_{0} \equiv H^{\prime}=18 \pi^{3} \alpha^{3} \frac{e^{2} a_{B}}{\lambda_{F}^{2}},
$$

\footnotetext{
${ }^{1}$ Of course, the electrons are quantum particles and their fixation cannot be considered too literally. Due to the Coulomb forces of ions, it is more favorable for collectivized electrons to be placed near the ions for the shielding of ions fields. At the same time, collectivized electrons are spread over whole metal. It is wrong to think that a particular electron is fixed inside a cell near to a particular ion. But the spread of the electrons does not play a fundamental importance for our further consideration, since there are two electrons near the node of the lattice in the divalent metal at any given time. They can be considered as located inside the cell as averaged.
} 
where $\alpha=\frac{1}{137}$ is the fine structure constant.

Comparing this association energy with the Fermi energy (12), we obtain

$$
\frac{\Delta_{0}}{\mathcal{E}_{F}}=9 \pi \alpha^{3} \simeq 1.1 \cdot 10^{-5}
$$

Assuming that the critical temperature below which the possible existence of such condensate is approximately equal

$$
T_{c} \simeq \frac{1}{2} \frac{\Delta_{0}}{k}
$$

(the coefficient approximately equal to $1 / 2$ corresponds to the experimental data, discussed below in the section (4.6)).

After substituting obtained parameters, we have

$$
T_{c} \simeq 5.5 \cdot 10^{-6} T_{F}
$$

The experimentally measured ratios $\frac{T_{c}}{T_{F}}$ for I-type superconductors are given in Table (1) and in Fig.(3).

The straight line on this figure is obtained from Eq.(20), which as seen defines an upper limit of critical temperatures of I-type superconductors.

\section{The condensate of zero-point oscillations and type-I super- conductors}

\subsection{The critical temperature of type-I superconductors}

In order to compare the critical temperature of the condensate of zero-point oscillations with measured critical temperatures of superconductors, at first we should make an estimation on the Fermi energies of superconductors. For this we use the experimental data for the Sommerfeld's constant through which the Fermi energy can be expressed:

$$
\gamma=\frac{\pi^{2} k^{2} n_{e}}{4 \mathcal{E}_{F}}=\frac{1}{2} \cdot\left(\frac{\pi}{3}\right)^{2 / 3}\left(\frac{k}{\hbar}\right)^{2} m_{e} n_{e}^{1 / 3}
$$

So on the basis of Eqs.(12) and (21), we get:

$$
k T_{F}(\gamma)=\frac{p_{F}^{2}(\gamma)}{2 m_{e}} \simeq\left(\frac{12}{k^{2}}\right)^{2}\left(\frac{\hbar^{2}}{2 m_{e}}\right)^{3} \gamma^{2} .
$$

On base of these calculations we obtain possibility to relate directly the critical temperature of a superconductor with the experimentally measurable parameter: with its electronic specific heat.

Taking into account Eq.(20), we have:

$$
\Delta_{0} \simeq \Theta \gamma^{2}
$$

where the constant

$$
\Theta \simeq 31 \frac{\pi^{2}}{k}\left[\frac{\alpha \hbar^{2}}{k m_{e}}\right]^{3} \simeq 6.65 \cdot 10^{-22} \frac{K^{4} \mathrm{~cm}^{6}}{\mathrm{erg}} .
$$

The comparison of the calculated parameters and measured data $([12],[11])$ is given in Table (1)-(2) and in Fig.(3) and (8).
Table 1. The comparison of the calculated values of superconductors critical temperatures with measured Fermi temperatures

\begin{tabular}{||c|c|c|c||}
\hline \hline superconductor & $T_{C}, \mathrm{~K}$ & $\begin{array}{c}T_{F}, \mathrm{~K} \\
\mathrm{Eq}(22)\end{array}$ & $\frac{T_{C}}{T_{F}}$ \\
\hline $\mathrm{Cd}$ & 0.51 & $1.81 \cdot 10^{5}$ & $2.86 \cdot 10^{-6}$ \\
$\mathrm{Zn}$ & 0.85 & $3.30 \cdot 10^{5}$ & $2.58 \cdot 10^{-6}$ \\
$\mathrm{Ga}$ & 1.09 & $1.65 \cdot 10^{5}$ & $6.65 \cdot 10^{-6}$ \\
$\mathrm{Tl}$ & 2.39 & $4.67 \cdot 10^{5}$ & $5.09 \cdot 10^{-6}$ \\
$\mathrm{In}$ & 3.41 & $7.22 \cdot 10^{5}$ & $4.72 \cdot 10^{-6}$ \\
$\mathrm{Sn}$ & 3.72 & $7.33 \cdot 10^{5}$ & $5.08 \cdot 10^{-6}$ \\
$\mathrm{Hg}$ & 4.15 & $1.05 \cdot 10^{6}$ & $3.96 \cdot 10^{-6}$ \\
$\mathrm{~Pb}$ & 7.19 & $1.85 \cdot 10^{6}$ & $3.90 \cdot 10^{-6}$ \\
\hline \hline
\end{tabular}

Table 2. The comparison of the calculated values of superconductors critical temperatures with measurement data

\begin{tabular}{||c|c|c|c|c||}
\hline \hline $\begin{array}{c}\text { super- } \\
\text { con- } \\
\text { ductors }\end{array}$ & $\begin{array}{c}T_{c}, \\
\text { (measur) } \\
\mathrm{K}\end{array}$ & $\begin{array}{c}\gamma, \\
\frac{e r g}{c m^{3} K^{2}}\end{array}$ & $\begin{array}{c}T_{c}, \mathrm{~K} \\
\text { (calc) } \\
\text { Eq. }(23)\end{array}$ & $\begin{array}{c}T_{c} \text { (calc) } \\
T_{c}(\text { meas })\end{array}$ \\
\hline $\mathrm{Cd}$ & 0.517 & 532 & 0.77 & 1.49 \\
$\mathrm{Zn}$ & 0.85 & 718 & 1.41 & 1.65 \\
$\mathrm{Ga}$ & 1.09 & 508 & 0.70 & 0.65 \\
$\mathrm{Tl}$ & 2.39 & 855 & 1.99 & 0.84 \\
$\mathrm{In}$ & 3.41 & 1062 & 3.08 & 0.90 \\
$\mathrm{Sn}$ & 3.72 & 1070 & 3.12 & 0.84 \\
$\mathrm{Hg}$ & 4.15 & 1280 & 4.48 & 1.07 \\
$\mathrm{~Pb}$ & 7.19 & 1699 & 7.88 & 1.09 \\
\hline \hline
\end{tabular}

\subsection{The relation of critical parameters of type-I superconductors}

The phenomenon of condensation of zero-point oscillations in the electron gas has its characteristic features.

There are several ways of destroying the zero-point oscillations condensate in electron gas:

Firstly, it can be evaporated by heating. In this case, evaporation of the condensate should possess the properties of an order-disorder transition.

Secondly, due to the fact that the oscillating electrons carry electric charge, the condensate can be destroyed by the application of a sufficiently strong magnetic field.

For this reason, the critical temperature and critical magnetic field of the condensate will be interconnected.

This interconnection should manifest itself through the relationship of the critical temperature and critical field of the superconductors, if superconductivity occurs as result of an ordering of zero-point fluctuations.

Let us assume that at a given temperature $T<T_{c}$ the system of vibrational levels of conducting electrons consists of only two levels:

firstly, basic level which is characterized by an antiphase oscillations of the electron pairs at the distance $\Lambda_{0} / 2$, and

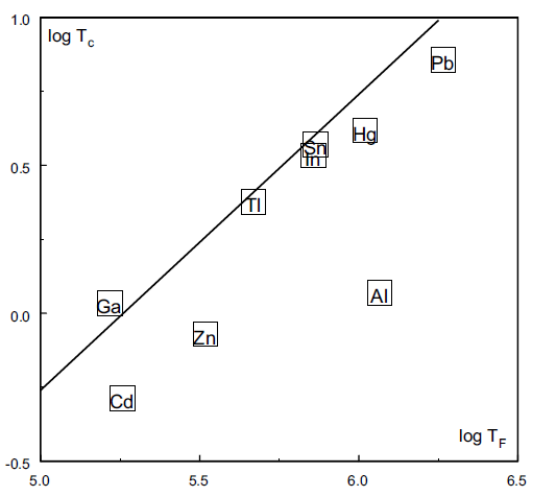

Figure 3. The comparison of critical temperatures $T_{c}$ of type-I superconductors with their Fermi temperatures $T_{F}$. The straight line is obtained from Eq. (20). 
secondly, an excited level characterized by in-phase oscillation of the pairs.

Let the population of the basic level be $N_{0}$ particles and the excited level has $N_{1}$ particles.

Two electron pairs at an in-phase oscillations have a high energy of interaction and therefore cannot form the condensate. The condensate can be formed only by the particles that make up the difference between the populations of levels $N_{0}-N_{1}$. In a dimensionless form, this difference defines the order parameter:

$$
\Psi=\frac{N_{0}}{N_{0}+N_{1}}-\frac{N_{1}}{N_{0}+N_{1}} .
$$

In the theory of superconductivity, by definition, the order parameter is determined by the value of the energy gap

$$
\Psi=\Delta_{T} / \Delta_{0}
$$

When taking a counting of energy from the level $\varepsilon_{0}$, we obtain

$$
\frac{\Delta_{T}}{\Delta_{0}}=\frac{N_{0}-N_{1}}{N_{0}+N_{1}} \simeq \frac{e^{2 \Delta_{T} / k T}-1}{e^{2 \Delta_{T} / k T}+1}=\operatorname{th}\left(2 \Delta_{T} / k T\right) .
$$

Passing to dimensionless variables $\delta \equiv \frac{\Delta_{T}}{\Delta_{0}}, t \equiv \frac{k T}{k T_{c}}$ and $\beta \equiv \frac{2 \Delta_{0}}{k T_{c}}$ we have

$$
\delta=\frac{e^{\beta \delta / t}-1}{e^{\beta \delta / t}+1}=\operatorname{th}(\beta \delta / t) .
$$

This equation describes the temperature dependence of the energy gap in the spectrum of zero-point oscillations. It is similar to other equations describing other physical phenomena, that are also characterized by the existence of the temperature dependence of order parameters [13],[14]. For example, this dependence is similar to temperature dependencies of the concentration of the superfluid component in liquid helium or the spontaneous magnetization of ferromagnetic materials. This equation is the same for all order-disorder transitions (the phase transitions of 2nd-type in the Landau classification).

The solution of this equation, obtained by the iteration method, is shown in Fig.(4).

This decision is in a agreement with the known transcendental equation of the BCS, which was obtained by the integration of the phonon spectrum, and is in a satisfactory agreement with the measurement data.

After numerical integrating we can obtain the averaging value of the gap:

$$
\langle\Delta\rangle=\Delta_{0} \int_{0}^{1} \delta d t=0.852 \Delta_{0} .
$$

To convert the condensate into the normal state, we must raise half of its particles into the excited state (according to Eq.(27), the gap collapses under this condition). To do this, taking into account Eq.(29), the unit volume of condensate should have the energy:

$$
\mathcal{E}_{T} \simeq \frac{1}{2} n_{0}\left\langle\Delta_{0}\right\rangle \approx \frac{0.85}{2}\left(\frac{m_{e}}{2 \pi^{2} \alpha \hbar^{2}}\right)^{3 / 2} \Delta_{0}^{5 / 2}
$$

On the other hand, we can obtain the normal state of an electrically charged condensate when applying a magnetic field of critical value $H_{c}$ with the density of energy:

$$
\mathcal{E}_{H}=\frac{H_{c}^{2}}{8 \pi}
$$

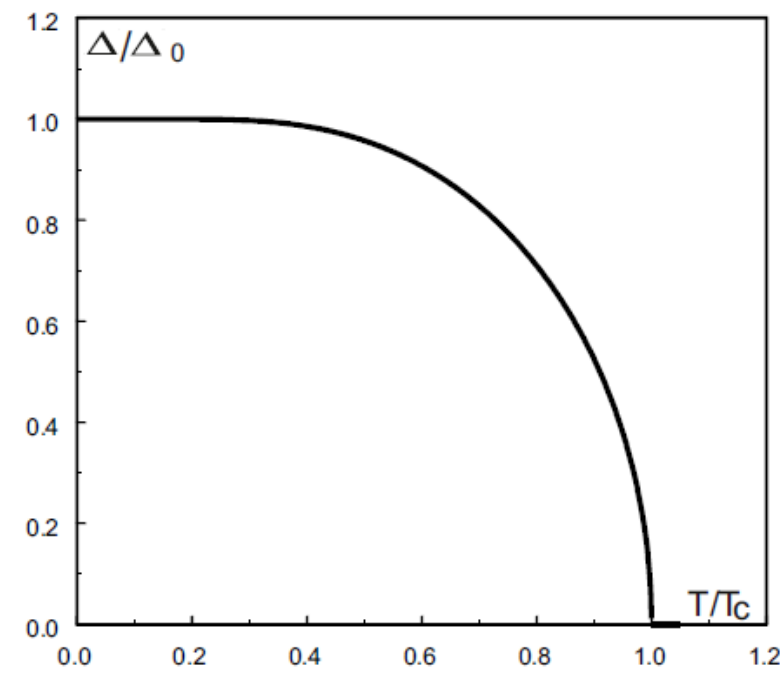

Figure 4. The temperature dependence of the value of the gap in the energetic spectrum of zero-point oscillations calculated on Eq.(28).

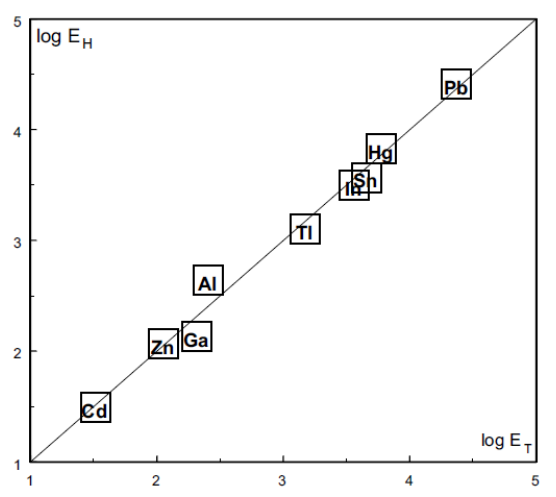

Figure 5. The comparison of the critical energy densities $\mathcal{E}_{T}$ (Eq.(30)) and $\mathcal{E}_{H}$ (Eq.(31)) for the type-I superconductors.

As a result, we acquire the condition:

$$
\frac{1}{2} n_{0}\left\langle\Delta_{0}\right\rangle=\frac{H_{c}^{2}}{8 \pi} .
$$

This creates a relation of the critical temperature to the critical magnetic field of the zero-point oscillations condensate of the charged bosons.

The comparison of the critical energy densities $\mathcal{E}_{T}$ and $\mathcal{E}_{H}$ for type-I superconductors are shown in Fig.(5). As shown, the obtained agreement between the energies $\mathcal{E}_{\mathcal{T}}$ (Eq.(30)) and $\mathcal{E}_{\mathcal{H}}$ (Eq.(31)) is quite satisfactory for typeI superconductors [11],[12]. A similar comparison for type-II superconductors shows results that differ by a factor two approximately. The reason for this will be considered below. The correction of this calculation, has not apparently made sense here. The purpose of these calculations was to show that the description of superconductivity as the effect of the condensation of ordered zero-point oscillations is in accordance with the available experimental data. This goal is considered reached in the simple case of type-I superconductors.

\subsection{The critical magnetic field of super- conductors}

The direct influence of the external magnetic field of the critical value applied to the electron system is 


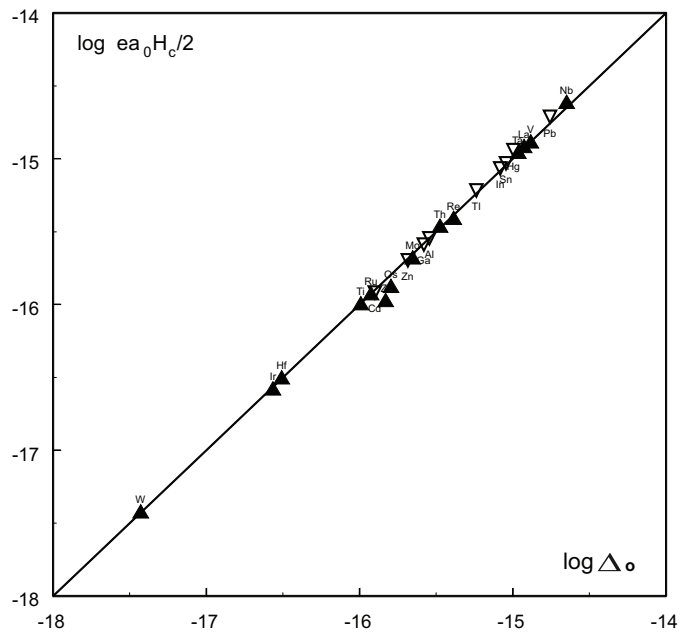

Figure 6. The comparison of the calculated energy of superconducting pairs in the critical magnetic field with the value of the superconducting gap. Here, the following key applies: filled triangles - type-II superconductors, empty triangles - type-I superconductors. On vertical axis - logarithm of the product of the calculated value of the oscillating dipole moment of an electron pair on the critical magnetic field is plotted.On horizontal axis the value of the gap is shown.

too weak to disrupt the dipole-dipole interaction of two paired electrons:

$$
\mu_{B} H_{c} \ll k T_{c} .
$$

In order to violate the superconductivity, the ordering of the electron zero-point oscillations must be destroyed. For this the presence of relatively weak magnetic field is required.

At combing of Eqs.(32),(30) and (16), we can express the gap through the critical magnetic field and the magnitude of the oscillating dipole moment:

$$
\Delta_{0} \approx \frac{1}{2} e a_{0} H_{c}
$$

The properties of the zero-point oscillations of the electrons should not be dependent on the characteristics of the mechanism of association and also on the condition of the existence of electron pairs. Therefore, we should expect that this equation would also be valid for type-I superconductors, as well as for II-type superconductors (for II-type superconductor $H_{c}=H_{c 1}$ is the first critical field)

An agreement with this condition is illustrated on the Fig.(6).

\subsection{The density of superconducting car- riers}

Let us consider the process of heating the electron gas in metal. When heating, the electrons from levels slightly below the Fermi-energy are raised to higher levels. As a result, the levels closest to the Fermi level, from which at low temperature electrons were forming bosons, become vacant.

At critical temperature $T_{c}$, all electrons from the levels of energy bands from $\mathcal{E}_{F}-\Delta$ to $\mathcal{E}_{F}$ move to higher levels (and the gap collapses). At this temperature superconductivity is therefore destroyed completely.
This band of energy can be filled by $N_{\Delta}$ particles:

$$
N_{\Delta}=2 \int_{\mathcal{E}_{F}-\Delta}^{\mathcal{E}_{F}} F(\mathcal{E}) D(\mathcal{E}) d \mathcal{E} .
$$

Where $F(\mathcal{E})=\frac{1}{e^{\frac{\mathcal{E}-\mu}{\tau}}+1}$ is the Fermi-Dirac function and $D(\mathcal{E})$ is number of states per an unit energy interval, a deuce front of the integral arises from the fact that there are two electron at each energy level.

To find the density of states $D(\mathcal{E})$, one needs to find the difference in energy of the system at $T=0$ and finite temperature:

$$
\Delta \mathcal{E}=\int_{0}^{\infty} F(\mathcal{E}) \mathcal{E} D(\mathcal{E}) d \mathcal{E}-\int_{0}^{\mathcal{E}_{F}} \mathcal{E} D(\mathcal{E}) d \mathcal{E} .
$$

For the calculation of the density of states $D(\mathcal{E})$, we must note that two electrons can be placed on each level. Thus, from the expression of the Fermi-energy Eq.(12) we obtain

$$
D\left(E_{F}\right)=\frac{1}{2} \cdot \frac{d n_{e}}{d \mathcal{E}_{F}}=\frac{3 n_{e}}{4 \mathcal{E}_{F}}=\frac{3 \gamma}{2 k^{2} \pi^{2}},
$$

where

$$
\gamma=\frac{\pi^{2} k^{2} n_{e}}{4 \mathcal{E}_{F}}=\frac{1}{2} \cdot\left(\frac{\pi}{3}\right)^{3 / 2}\left(\frac{k}{\hbar}\right)^{2} m_{e} n_{e}^{1 / 3}
$$

is the Sommerfeld constant ${ }^{2}$.

Using similar arguments, we can calculate the number of electrons, which populate the levels in the range from $\mathcal{E}_{F}-\Delta$ to $\mathcal{E}_{F}$. For an unit volume of material, Eq.(35) can be rewritten as:

$$
n_{\Delta}=2 k T \cdot D\left(\mathcal{E}_{F}\right) \int_{-\frac{\Delta_{0}}{k T_{c}}}^{0} \frac{d x}{\left(e^{x}+1\right)} .
$$

By supposing that for superconductors $\frac{\Delta_{0}}{k T_{c}}=1.86$, as a result of numerical integration we obtain

$$
\int_{-\frac{\Delta_{0}}{k T_{c}}}^{0} \frac{d x}{\left(e^{x}+1\right)}=\left[x-\ln \left(e^{x}+1\right)\right]_{-1.86}^{0} \approx 1.22 .
$$

Thus, the density of electrons, which throw up above the Fermi level in a metal at temperature $T=T_{c}$ is

$$
n_{e}\left(T_{c}\right) \approx 2.44\left(\frac{3 \gamma}{k^{2} \pi^{2}}\right) k T_{c}
$$

Where the Sommerfeld constant $\gamma$ is related to the volume unit of the metal.

From Eq.(6) it follows

$$
L_{0} \simeq \frac{\lambda_{F}}{\pi \alpha}
$$

and this forms the ratio of the condensate particle density to the Fermi gas density:

$$
\frac{n_{0}}{n_{e}}=\frac{\lambda_{F}^{3}}{L_{0}^{3}} \simeq(\pi \alpha)^{3} \simeq 10^{-5} .
$$

\footnotetext{
${ }^{2}$ It should be noted that because on each level two electrons can be placed, the expression for the Sommerfeld constant Eq.(38) contains the additional factor $1 / 2$ in comparison with the usual formula in literature [14]
} 
Table 3. The comparison of the superconducting carriers density at $T=0$ with the density of thermally activated electrons at $T=T_{c}$

\begin{tabular}{||c|c|c|c||}
\hline \hline $\begin{array}{c}\text { super- } \\
\text { con- } \\
\text { ductor }\end{array}$ & $n_{0}$ & $n_{e}\left(T_{c}\right)$ & $\frac{2 n_{0}}{n_{e}\left(T_{c}\right)}$ \\
\hline $\mathrm{Cd}$ & $6.11 \cdot 10^{17}$ & $1.48 \cdot 10^{18}$ & 0.83 \\
$\mathrm{Zn}$ & $1.29 \cdot 10^{18}$ & $3.28 \cdot 10^{18}$ & 0.78 \\
$\mathrm{Ga}$ & $1.85 \cdot 10^{18}$ & $2.96 \cdot 10^{18}$ & 1.25 \\
$\mathrm{Al}$ & $2.09 \cdot 10^{18}$ & $8.53 \cdot 10^{18}$ & 0.49 \\
$\mathrm{Tl}$ & $6.03 \cdot 10^{18}$ & $1.09 \cdot 10^{19}$ & 1.10 \\
$\mathrm{In}$ & $1.03 \cdot 10^{19}$ & $1.94 \cdot 10^{19}$ & 1.06 \\
$\mathrm{Sn}$ & $1.18 \cdot 10^{19}$ & $2.14 \cdot 10^{19}$ & 1.10 \\
$\mathrm{Hg}$ & $1.39 \cdot 10^{19}$ & $2.86 \cdot 10^{19}$ & 0.97 \\
$\mathrm{~Pb}$ & $3.17 \cdot 10^{19}$ & $6.58 \cdot 10^{19}$ & 0.96 \\
\hline \hline
\end{tabular}

When using these equations, we can find a linear dimension of localization for an electron pair:

$$
L_{0}=\frac{\Lambda_{0}}{2} \simeq \frac{1}{\pi \alpha\left(n_{e}\right)^{1 / 3}} .
$$

or, taking into account Eq.(16), we can obtain the relation between the density of particles in the condensate and the value of the energy gap:

$$
\Delta_{0} \simeq 2 \pi^{2} \alpha \frac{\hbar^{2}}{m_{e}} n_{0}^{2 / 3}
$$

or

$$
n_{0}=\frac{1}{L_{0}^{3}}=\left(\frac{m_{e}}{2 \pi^{2} \alpha \hbar^{2}} \Delta_{0}\right)^{3 / 2} .
$$

It should be noted that the obtained ratios for the zero-point oscillations condensate (of bose-particles) differ from the corresponding expressions for the bosecondensate of particles, which can be obtained in many courses (see eg [13]). The expressions for the ordered condensate of zero-point oscillations have an additional coefficient $\alpha$ on the right side of Eq.(45).

The de Broglie wavelengths of Fermi electrons expressed through the Sommerfelds constant

$$
\lambda_{F}=\frac{2 \pi \hbar}{p_{F}(\gamma)} \simeq \frac{\pi}{3} \cdot \frac{k^{2} m_{e}}{\hbar^{2} \gamma}
$$

are shown in Tab.3.

In accordance with Eq.(42), which was obtained at the zero-point oscillations consideration, the ratio $\frac{\lambda_{F}}{\Lambda_{0}} \simeq$ $2.3 \cdot 10^{-2}$.

In connection with this ratio, the calculated ratio of the zero-point oscillations condensate density to the density of fermions in accordance with Eq.(43) should be near to $10^{-5}$.

It can be therefore be seen, that calculated estimations of the condensate parameters are in satisfactory agreement with experimental data of superconductors.

Based on these calculations, it is interesting to compare the density of superconducting carriers $n_{0}$ at $T=0$, which is described by Eq.(46), with the density of normal carriers $n_{e}\left(T_{c}\right)$, which are evaporated on levels above $\mathcal{E}_{F}$ at $T=T_{c}$ and are described by Eq.(41).
Table 4. The ratios $\frac{\lambda_{F}}{\Lambda_{0}}$ and $\frac{n_{0}}{n_{e}}$ for type-I superconductors

\begin{tabular}{||c|c|c|c|c||}
\hline \hline $\begin{array}{c}\text { super- } \\
\text { con- } \\
\text {-ductor }\end{array}$ & $\begin{array}{c}\lambda_{F}, \\
10^{-8} \mathrm{~cm} \\
\mathrm{Eq}(47)\end{array}$ & $\begin{array}{c}\Lambda_{0}, \\
10^{-6} \mathrm{~cm} \\
\mathrm{Eq}(6)\end{array}$ & $\frac{\lambda_{F}}{\Lambda_{0}}$ & $\frac{n_{0}}{n_{e}}=\left(\frac{\lambda_{F}}{\Lambda_{0}}\right)^{3}$ \\
\hline $\mathrm{Cd}$ & 3.1 & 1.18 & $2.6 \cdot 10^{-2}$ & $1.8 \cdot 10^{-5}$ \\
$\mathrm{Zn}$ & 2.3 & 0.92 & $2.5 \cdot 10^{-2}$ & $1.5 \cdot 10^{-5}$ \\
$\mathrm{Ga}$ & 3.2 & 0.81 & $3.9 \cdot 10^{-2}$ & $6.3 \cdot 10^{-5}$ \\
$\mathrm{Tl}$ & 1.9 & 0.55 & $3.4 \cdot 10^{-2}$ & $4.3 \cdot 10^{-5}$ \\
$\mathrm{In}$ & 1.5 & 0.46 & $3.2 \cdot 10^{-2}$ & $3.8 \cdot 10^{-5}$ \\
$\mathrm{Sn}$ & 1.5 & 0.44 & $3.4 \cdot 10^{-2}$ & $4.3 \cdot 10^{-5}$ \\
$\mathrm{Hg}$ & 1.3 & 0.42 & $3.1 \cdot 10^{-2}$ & $2.9 \cdot 10^{-5}$ \\
$\mathrm{~Pb}$ & 1.0 & 0.32 & $3.1 \cdot 10^{-2}$ & $2.9 \cdot 10^{-5}$ \\
\hline \hline
\end{tabular}

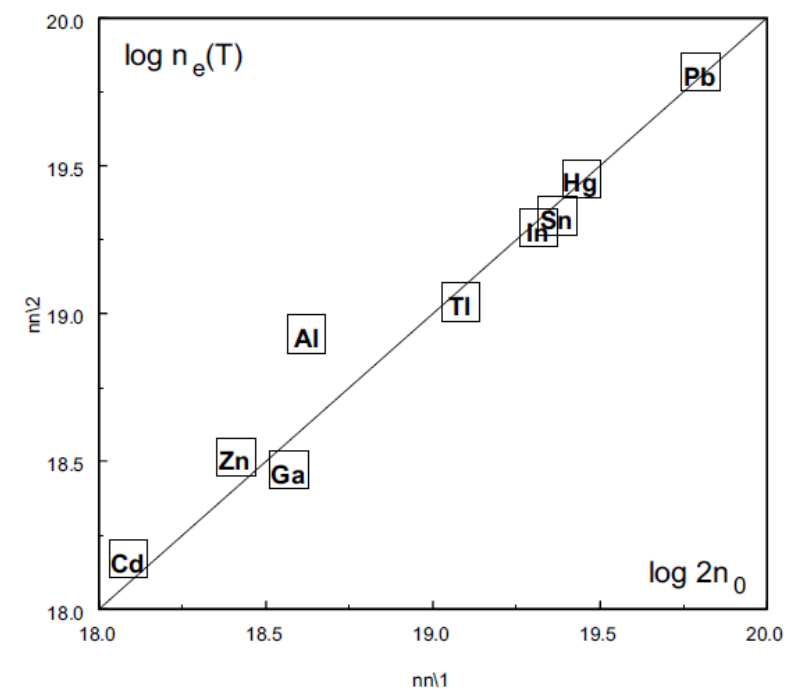

Figure 7. The comparison of the number of superconducting carriers at $T=0$ with the number of thermally activated electrons at $T=T_{c}$.

This comparison is shown in Table (3) and Fig.7. (Data has been taken from the tables [11], [12]).

From the data described above, we can obtain the condition of destruction of superconductivity, after heating for superconductors of type-I, as written in the equation:

$$
n_{e}\left(T_{c}\right) \simeq 2 n_{0}
$$

\subsection{The sound velocity of the zero-point oscillations condensate}

The wavelength of zero-point oscillations $\Lambda_{0}$ in this model is an analogue of the Pippard coherence length in the BCS. As usually accepted [12], the coherence length $\xi=\frac{\hbar v_{F}}{4 \Delta_{0}}$. The ratio of these lengths, taking into account Eq. (20), is simply the constant:

$$
\frac{\Lambda_{0}}{\xi} \approx 8 \pi^{2} \alpha^{2} \approx \cdot 10^{-3}
$$

The attractive forces arising between the dipoles located at a distance $\frac{\Lambda_{0}}{2}$ from each other and vibrating in opposite phase, create pressure in the system:

$$
P \simeq \frac{d \Delta_{0}}{d V} \simeq \frac{d_{\Omega}^{2}}{L_{0}^{6}}
$$

In this regard, sound into this condensation should propagate with the velocity:

$$
c_{s} \simeq \sqrt{\frac{1}{2 m_{e}} \frac{d P}{d n_{0}}} .
$$


Table 5. The value of ratio $\Delta_{0} / k T_{c}$ obtained experimentally for type-I superconductors

\begin{tabular}{||c|c|c|c||}
\hline \hline superconductor & $T_{c}, \mathrm{~K}$ & $\Delta_{0}, \mathrm{mev}$ & $\frac{\Delta_{0}}{k T_{c}}$ \\
\hline $\mathrm{Cd}$ & 0.51 & 0.072 & 1.64 \\
$\mathrm{Zn}$ & 0.85 & 0.13 & 1.77 \\
$\mathrm{Ga}$ & 1.09 & 0.169 & 1.80 \\
$\mathrm{Tl}$ & 2.39 & 0.369 & 1.79 \\
$\mathrm{In}$ & 3.41 & 0.541 & 1.84 \\
$\mathrm{Sn}$ & 3.72 & 0.593 & 1.85 \\
$\mathrm{Hg}$ & 4.15 & 0.824 & 2.29 \\
$\mathrm{~Pb}$ & 7.19 & 1.38 & 2.22 \\
\hline \hline
\end{tabular}

After the appropriate substitutions, the speed of sound in the condensate can be expressed through the Fermi velocity of electron gas

$$
c_{s} \simeq \sqrt{2 \pi^{2} \alpha^{3}} v_{F} \simeq 10^{-2} v_{F} .
$$

The condensate particles moving with velocity $c_{S}$ have the kinetic energy:

$$
2 m_{e} c_{s}^{2} \simeq \Delta_{0}
$$

Therefore, by either heating the condensate to the critical temperature when each of its volume obtains the energy $\mathcal{E} \approx n_{0} \Delta_{0}$, or initiating the current of its particles with a velocity exceeding $c_{S}$, can achieve the destruction of the condensate. (Because the condensate of charged particles oscillations is considered, destroying its coherence can be also obtained at the application of a sufficiently strong magnetic field. See below.)

\subsection{The relationship $\Delta_{0} / k T_{c}$}

From Eq.(48) and taking into account Eqs.(23),(41) and (46), which were obtained for condensate, we have:

$$
\frac{\Delta_{0}}{k T_{c}} \simeq 1.86
$$

This estimation of the relationship $\Delta_{0} / k T_{c}$ obtained for condensate has a satisfactory agreement with the measured data [11], for type-I superconductors as listed in Table (5). ${ }^{3}$

\section{The estimation of properties of type-II superconductors}

In the case of type-II superconductors the situation is more complicated.

In this case, measurements show that these metals have an electronic specific heat that has an order of value greater than those calculated on the base of free electron gas model.

The peculiarity of these metals is associated with the specific structure of their ions. They are transition metals with unfilled inner d-shell (see Table 6).

It can be assumed that the increase in the electronic specific heat of these metals should be associated with a
Table 6. The external electron shells of elementary type-II superconductors

\begin{tabular}{||c|c||}
\hline \hline superconductors & electron shells \\
\hline$T i$ & $3 d^{2} 4 s^{2}$ \\
$V$ & $3 d^{3} 4 s^{2}$ \\
$Z r$ & $4 d^{2} 5 s^{2}$ \\
$N b$ & $4 d^{3} 5 s^{2}$ \\
$M o$ & $4 d^{4} 5 s^{2}$ \\
$T c$ & $4 d^{5} 5 s^{2}$ \\
$R u$ & $4 d^{6} 5 s^{2}$ \\
$L a$ & $5 d^{1} 6 s^{2}$ \\
$H f$ & $5 d^{2} 6 s^{2}$ \\
$T a$ & $5 d^{3} 6 s^{2}$ \\
$W$ & $5 d^{4} 6 s^{2}$ \\
$R e$ & $5 d^{5} 6 s^{2}$ \\
$O s$ & $5 d^{6} 6 s^{2}$ \\
$I r$ & $5 d^{7} 6 s^{2}$ \\
\hline \hline
\end{tabular}

characteristic interaction of free electrons with the electrons of the unfilled d-shell.

Since the heat capacity of the ionic lattice of metals is negligible at low temperatures, only the electronic subsystem is thermally active .

At $T=0$ the superconducting careers populates the energetic level $\mathcal{E}_{F}-\Delta_{0}$. During the destruction of superconductivity through heating, an each heated career increases its thermal vibration. If the effective velocity of vibration is $v_{t}$, its kinetic energy:

$$
\mathcal{E}_{k}=\frac{m v_{t}^{2}}{2} \simeq \Delta_{0}
$$

Only a fraction of the heat energy transferred to the metal is consumed in order to increase the kinetic energy of the electron gas in the transition metals.

Another part of the energy will be spent on the magnetic interaction of a moving electron.

At contact with the d-shell electron, a freely moving electron induces onto it the magnetic field of the order of value:

$$
H \approx \frac{e}{r_{c}^{2}} \frac{v}{c} .
$$

The magnetic moment of d-electron is approximately equal to the Bohr magneton. Therefore the energy of the magnetic interaction between a moving electron of conductivity and a d-electron is approximately equal to:

$$
\mathcal{E}_{\mu} \approx \frac{e^{2}}{2 r_{c}} \frac{v}{c}
$$

This energy is not connected with the process of destruction of superconductivity.

Whereas, in metals with a filled d-shell (type-I superconductors), the whole heating energy increases the kinetic energy of the conductivity electrons and only a small part of the heating energy is spent on it in transition metals:

$$
\frac{\mathcal{E}_{k}}{\mathcal{E}_{\mu}+\mathcal{E}_{k}} \simeq \frac{m v_{t}}{h} a_{B}
$$

So approximately

$$
\frac{\mathcal{E}_{k}}{\mathcal{E}_{\mu}+\mathcal{E}_{k}} \simeq \frac{a_{B}}{L_{0}}
$$

\footnotetext{
${ }^{3}$ In the BCS-theory $\frac{\Delta_{0}}{k T_{c}} \simeq 1.76$.
} 


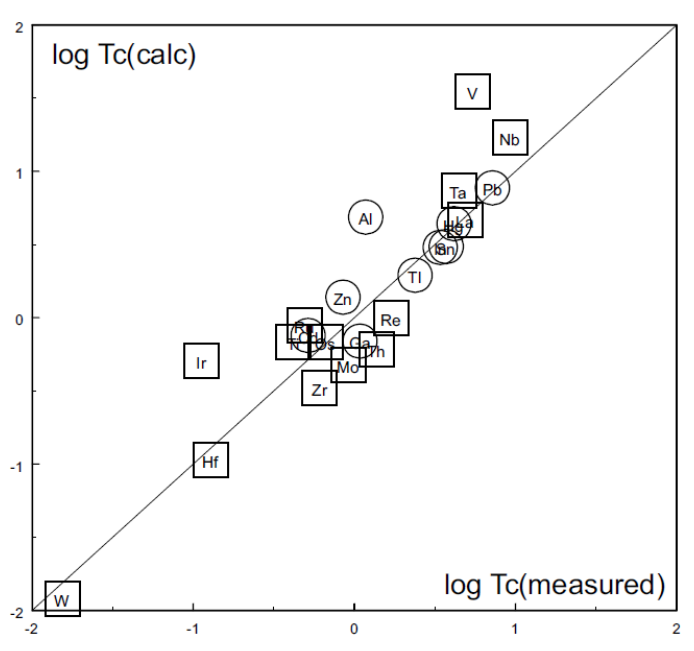

Figure 8. The comparison of the calculated values of critical temperatures of superconductors with measurement data. Circles relate to type-I superconductors, squares show type-II superconductors. On the abscissa, the measured values of critical temperatures are plotted, on ordinate, the calculated estimations are plotted. The calculations of critical temperatures for type-I superconductors were made by using Eq.(23) and the estimations for type-II superconductors was obtained by using Eq.(60).

Therefore, whereas the dependence of the gap in typeI superconductors from the heat capacity is defined by Eq.(23), it is necessary to take into account the relation Eq.(59) in type-II superconductors for the determination of this gap dependence. As a result of this estimation, we can obtain:

$$
\Delta_{0} \simeq \Theta \gamma^{2}\left(\frac{\mathcal{E}_{k}}{\mathcal{E}_{\mu}+\mathcal{E}_{k}}\right) \simeq \Theta \gamma^{2}\left(\frac{a_{B}}{L_{0}}\right) \frac{1}{2},
$$

where $1 / 2$ is the fitting parameter.

The comparison of the results of these calculations with the measurement data (Fig.(8)) shows that for the majority of type-II superconductors the estimation Eq.(60) can be considered quite satisfactory. ${ }^{4}$

\section{Alloys and high-temperature superconductors}

In order to understand the mechanism of high temperature superconductivity, it is important to establish whether the high- $T_{c}$ ceramics are the I or II-type superconductors, or whether they are a special class of superconductors.

In order to determine this, we need to look at the above established dependence of critical parameters from the electronic specific heat and also consider that

\footnotetext{
${ }^{4}$ The lowest critical temperature was measured for $\mathrm{Mg}$. It is approximately equal to $1 \mathrm{mK}$. Mg-atoms in the metallic state are given two electrons into the electron gas of conductivity. It is confirmed by the fact that the pairing of these electrons, which manifests itself in the measured value of the flux quantum [9], is observed above $T_{c}$. It would seem that in view of this metallic Mg-ion must have electron shell like the Ne-atom. Therefore it is logical to expect that the critical temperature of $\mathrm{Mg}$ can be calculated by the formula for I-type superconductors. But actually in order to get the value of $T_{c} \approx 1 \mathrm{mK}$, the critical temperature of $\mathrm{Mg}$ should be calculated by the formula (60), which is applicable to the description of metals with an unfilled inner shell. This suggests that the ionic core of magnesium metal apparently is not as simple as the completely filled Ne-shell.
}

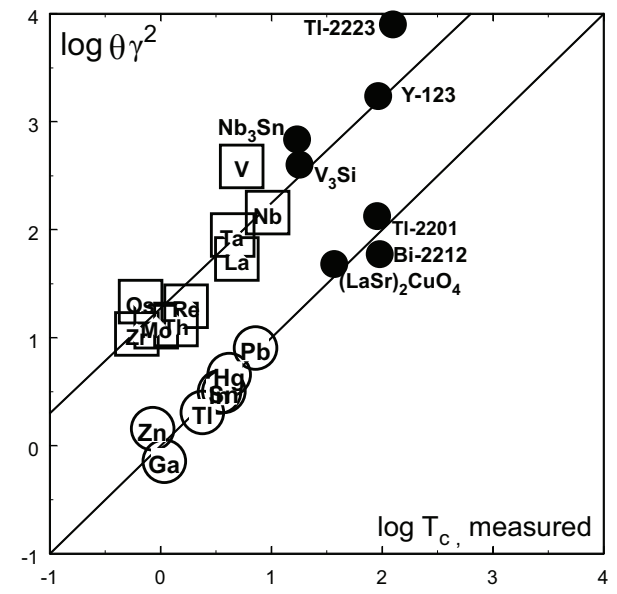

Figure 9. The comparison of the calculated parameter $\Theta \gamma^{2}$ with the measurement of the critical temperatures of elementary superconductors and some superconducting compounds.

the specific heat of superconductors I and II-types are differing considerably.

There are some difficulties by determining the answer this way: as we do not precisely know the density of the electron gas in high-temperature superconductors. However, the densities of atoms in metals do not differ too much and we can use Eq.(23) for the solution of the problem of the I- and II-types superconductors distinguishing.

If parameters of type-I superconductors are inserted into this equation, we obtain quite a satisfactory estimation of the critical temperature (as was done above, see Fig.8). For the type-II superconductors' values, this assessment gives an overestimated value due to the fact that type-II superconductors' specific heat has additional term associated with the magnetization of $d-$ electrons.

This analysis therefore, illustrates a possibility where we can divide all superconductors into two groups, as is evident from the Fig.(9).

It is generally assumed that we consider alloys $\mathrm{Nb}_{3} \mathrm{Sn}$ and $V_{3} S i$ as the type-II superconductors. This assumption seems quite normal because they are placed in close surroundings of Nb. Some excess of the calculated critical temperature over the experimentally measured value for ceramics $\mathrm{Ta}_{2} \mathrm{Ba}_{2} \mathrm{Ca}_{2} \mathrm{Cu}_{3} \mathrm{O}_{10}$ can be attributed to the measured heat capacity that may have been created by not only conductive electrons, but also non-superconducting elements (layers) of ceramics. It is already known that it, as well as ceramics $\mathrm{YBa}_{2} \mathrm{Cu}_{3} \mathrm{O}_{7}$, belongs to the type-II superconductors. However, ceramics (LaSr) ${ }_{2} \mathrm{Cu}_{4}, \mathrm{Bi}-2212$ and Tl-2201, according to this figure should be regarded as type-I superconductors, which is unusual.

\section{About the London penetration depth}

\subsection{The magnetic energy of a moving electron}

To avoid these incorrect results, let us consider a balance of magnetic energy in a superconductor within 
magnetic field. This magnetic energy is composed of energy from a penetrating external magnetic field and magnetic energy of moving electrons.

By using of the standard formulas of electrodynamics [15], let us estimate the ratio of the magnetic and kinetic energy of the electron (the charge of $e$ and the mass $m_{e}$ ) while moving rectilinearly with a velocity $v \ll c$.

The density of the electromagnetic field momentum is expressed by the equation:

$$
\mathbf{g}=\frac{1}{4 \pi c}[\mathbf{E H}]
$$

While moving with a velocity $\mathbf{v}$, the electric charge carrying the electric field with intensity $E$ creates a magnetic field

$$
\mathbf{H}=\frac{1}{c}[\mathbf{E v}]
$$

with the density of the electromagnetic field momentum (at $v \ll c)$

$$
\mathbf{g}=\frac{1}{4 \pi c^{2}}[\mathbf{E}[\mathbf{v E}]]=\frac{1}{4 \pi c^{2}}\left(\mathbf{v} E^{2}-\mathbf{E}(\mathbf{v} \cdot \mathbf{E})\right)
$$

As a result, the momentum of the electromagnetic field of a moving electron

$$
\begin{aligned}
& \mathbf{G}=\int_{V} \mathbf{g} d V= \\
& =\frac{1}{4 \pi c^{2}}\left(\mathbf{v} \int_{V} E^{2} d V-\int_{V} \mathbf{E} E v \cos \vartheta d V\right)
\end{aligned}
$$

The integrals are taken over the entire space, which is occupied by particle fields, and $\vartheta$ is the angle between the particle velocity and the radius vector of the observation point. By calculating the last integral in the condition of the axial symmetry with respect to $\mathbf{v}$, the contributions from the components of the vector $\mathbf{E}$, which is perpendicular to the velocity, cancel each other for all pairs of elements of the space (if they located diametrically opposite on the magnetic force line). Therefore, according to Eq.(64), the component of the field which is collinear to $\mathbf{v}$

$$
\frac{E \cos \vartheta \cdot \mathbf{v}}{v}
$$

can be taken instead of the vector $\mathbf{E}$. By taking this information into account, going over to the spherical coordinates and integrating over angles, we can obtain

$$
\mathbf{G}=\frac{\mathbf{v}}{4 \pi c^{2}} \int_{r}^{\infty} E^{2} \cdot 4 \pi r^{2} d r
$$

If we limit the integration of the field by the Compton electron radius $r_{C}=\frac{\hbar}{m_{e} c}, \quad 5$ then $v \ll c$, we can obtain:

$$
\mathbf{G}=\frac{\mathbf{v}}{4 \pi c^{2}} \int_{r_{C}}^{\infty} E^{2} \cdot 4 \pi r^{2} d r=\frac{\mathbf{v}}{c^{2}} \frac{e^{2}}{r_{C}}
$$

In this case by taking into account Eq.(62), the magnetic energy of a slowly moving electron pair is equal to:

$$
\mathcal{E}=\frac{v G}{2}=\frac{v^{2}}{c^{2}} \frac{e^{2}}{2 r_{C}}=\alpha \frac{m_{e} v^{2}}{2} .
$$

\footnotetext{
${ }^{5}$ Such effects as the pair generation force us to consider the radius of the "quantum electron" as approximately equal to Compton radius [16].
}

\subsection{The magnetic energy and the Lon- don penetration depth}

The energy of external magnetic field into volume $d v$ :

$$
\mathcal{E}=\frac{H^{2}}{8 \pi} d v
$$

At a density of superconducting carriers $n_{s}$, their magnetic energy per unit volume in accordance with (68):

$$
\mathcal{E}_{H} \simeq \alpha n_{s} \frac{m_{2} v^{2}}{2}=\alpha \frac{m_{e} j_{s}^{2}}{2 n_{s} e},
$$

where $j_{s}=2 e n_{s} v_{s}$ is the density of a current of superconducting carriers.

Taking into account the Maxwell equation

$$
\operatorname{rot} \mathbf{H}=\frac{4 \pi}{c} \mathbf{j}_{s},
$$

the magnetic energy of moving carriers can be written as

$$
\mathcal{E}_{H} \simeq \frac{\widetilde{\Lambda}^{2}}{8 \pi}(\operatorname{rot} H)^{2},
$$

where we introduce the notation

$$
\widetilde{\Lambda}=\sqrt{\alpha \frac{m_{e} c^{2}}{4 \pi n_{s} e^{2}}}=\sqrt{\alpha} \Lambda_{L} .
$$

In this case, part of the free energy of the superconductor connected with the application of a magnetic field is equal to:

$$
\mathcal{F}_{H}=\frac{1}{8 \pi} \int_{V}\left(H^{2}+\widetilde{\Lambda}^{2}(\operatorname{rot} H)^{2}\right) d v .
$$

At the minimization of the free energy, after some simple transformations we obtain

$$
\mathbf{H}+\widetilde{\Lambda}^{2} \operatorname{rotrot} \mathbf{H}=0,
$$

thus $\widetilde{\Lambda}$ is the depth of magnetic field penetration into the superconductor.

In view of Eq.(46) from Eq.(73) we can estimate the values of London penetration depth (see table (7.2)). The consent of the obtained values with the measurement data can be considered quite satisfactory.

Table 7.2
\begin{tabular}{||c|c|c|c||}
\hline $\begin{array}{c}\text { super- } \\
\text { conductors }\end{array}$ & $\begin{array}{c}\lambda_{L}, 10^{-6} \mathrm{~cm} \\
\text { measured }[17]\end{array}$ & $\begin{array}{c}\widetilde{\Lambda}, 10^{-6} \mathrm{~cm} \\
\text { calculated } \\
\text { Eq.(73) }\end{array}$ & $\widetilde{\Lambda} / \lambda_{L}$ \\
\hline $\mathrm{Tl}$ & 9.2 & 11.0 & 1.2 \\
$\mathrm{In}$ & 6.4 & 8.4 & 1.3 \\
$\mathrm{Sn}$ & 5.1 & 7.9 & 1.5 \\
$\mathrm{Hg}$ & 4.2 & 7.2 & 1.7 \\
$\mathrm{~Pb}$ & 3.9 & 4.8 & 1.2 \\
\hline
\end{tabular}

The resulting refinement may be important for estimates within the frame of Ginzburg-Landau theory, where the London penetration depth is used as a comparison of calculations and specific parameters of superconductors. 


\section{Three words to experimenters}

\subsection{Why a creation of room- temperature superconductors are hardly probably?}

The understanding of the mechanism of the superconducting state should open a way towards finding a solution to the technological problem. This problem was just a dream in the last century - the dream to fabricate a superconductor that was easily produced (in the sense of ductility) and had high critical temperature.

In order to move towards achieving this goal, it important to firstly understand the mechanism that limits of the critical properties of superconductors.

Let us consider a superconductor with a large limiting current. The length of their localisation determines the limiting momentum of superconducting carriers:

$$
p_{c} \simeq \frac{2 \pi \hbar}{L_{0}} .
$$

Therefore, by using the Eq.(53), we can compare the critical velocity of superconducting carriers with the sound velocity:

$$
v_{c}=\frac{p_{c}}{2 m_{e}} \simeq c_{s}
$$

and both these velocities are about a hundred times smaller than the Fermi velocity.

The sound velocity in the crystal lattice of metal $v_{s}$, in accordance with the Bohm-Staver relation [19], has approximately the same value:

$$
v_{s} \simeq \frac{k T_{D}}{E_{F}} v_{F} \simeq 10^{-2} v_{F} .
$$

This therefore, makes it possible to consider superconductivity being destroyed as a superconducting carrier overcomes the sound barrier. That is, if they moved without friction at a speed that was less than that of sound, after it gained speed and the speed of sound was surpassed, it then acquire a mechanism of friction.

Therefore, it is conceivable that if the speed of sound in the metal lattice $v_{s}<c_{s}$, then it would create a restriction on the limiting current in the superconductor.

If this is correct, then superconductors with high critical parameters should have not only a high Fermi energy of their electron gas, but also have a high speed of sound in their lattice.

It is in agreement with the fact that ceramics have higher elastic moduli compared to metals and alloys and also poss much higher critical temperatures (Fig.10).

The dependence of the critical temperature on the square of the speed of sound [18] is illustrated in Fig.(10).

This figure, which can be viewed only as a rough estimation due to the lack of necessary experimental data, shows that the elastic modulus of ceramics with a critical temperature close to room temperature should be close to the elastic modulus of sapphire, which is very difficult to achieve.

In addition, such ceramics would be deprived of yet an other important quality - their adaptability. Indeed,

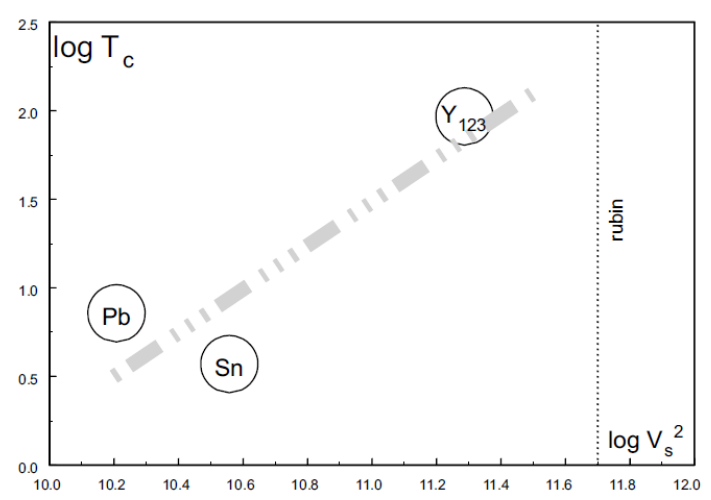

Figure 10. The schematic representation of the dependence of critical temperature on the speed of sound in superconductors. On the ordinate, the logarithm of the critical temperature of superconductor is shown. On the abscissa, the logarithm of the square of the speed of sound is shown (for $\mathrm{Sn}$ and $\mathrm{Pb}$ - the transverse velocity of sound is shown, because it is smaller. The speed of sound in a film was used for yttrium-123 ceramics. The dashed line shows the value of the transverse velocity of sound in sapphire, as some estimation of the limit of its value. It can be seen that this estimation leads to the restriction on the critical temperature in the range of $0^{\circ} \mathrm{C}$ - the dot-dashed line.

in order to obtain a thin wire, we require a plastic superconductor.

A solution of this problem would be to find a material that possesses an acceptably high critical temperature (above $80 \mathrm{~K}$ ) and also experiences a phase transition at an even higher temperature of heat treatment. It would be possible to make a thin wire from a superconductor near the point of phase transition, as the elastic modules are typically not usually very strong at this stage.

\subsection{Magnetic electron pairing}

This considered formation of mechanism for the superconducting state provides a possibility of obtaining the estimations of the critical parameters of superconductors, which in most cases is in satisfactory agreement with measured data. For some superconductors, this agreement is stronger, and for other, such as Ir, Al, V (see Fig.(8)), it is expedient to carry out further theoretical and experimental studies due to causes of deviations.

The mechanism of magnetic electron pairing is also of fundamental interest in order to further clarify this.

As was found earlier, in the cylinders made from certain superconducting metals $(\mathrm{Al}[8]$ and $\mathrm{Mg}[9])$, the observed magnetic flux quantization has exactly the same period above $T_{c}$ and that below $T_{c}$. The authors of these studies attributed this to the influence of a special effect. It seems more natural to think that the stability of the period is a result of the pairing of electrons due to magnetic dipole-dipole interaction continuing to exist at temperatures above $T_{c}$, despite the disappearance of the material's superconducting properties. At this temperature the coherence of the zero-point fluctuations is destroyed, and with it so is the superconductivity.

The pairing of electrons due to dipole-dipole interaction should be absent in the monovalent metals. In these metals, the conduction electrons are localized in the lattice at very large distances from each other.

It is therefore interesting to compare the period of quantization in the two cases. In a thin cylinder made 
of a superconductor, such as $\mathrm{Mg}$, above $T_{c}$ the quantization period is equal to $\frac{2 \pi \hbar c}{2 e}$. In the same cylinder of a noble metal (such as gold), the sampling period should be twice as large.

\subsection{The effect of isotopic substitution on the condensation of zero-point oscil- lations}

The attention of experimentalists could be attracted to the isotope effect in superconductors, which served as a starting point of the BCS theory. In the '50s it had been experimentally established that there is a dependence of the critical temperature of superconductors due to the mass of the isotope. Because the effect depends on the ion mass, this is considered to be because of the fact that it is based on the vibrational (phonon) process.

The isotope effect for a number of I-type superconductors - $\mathrm{Zn}, \mathrm{Sn}, \mathrm{In}, \mathrm{Hg}, \mathrm{Pb}$ - can be described by the relationship:

$$
\sqrt{M_{i}} T_{c}=\text { const }
$$

where $M_{i}$ - the mass of the isotope, $T_{c}$ is the critical temperature. The isotope effect in other superconductors can either be described by other dependencies, or is absent altogether.

In recent decades, however, the effects associated with the replacement of isotopes in the metal lattice have been studied in detail. It was shown that the zero-point oscillations of ions in the lattice of many metals are nonharmonical. Therefore, the isotopic substitution can directly affect the lattice parameters, the density of the lattice and the density of the electron gas in the metal, on its Fermi energy and on other properties of the electron subsystem.

The direct study of the effect of isotopic substitution on the lattice parameters of superconducting metals has not been carried out.

The results of measurements made on $G e, S i$, diamond and light metals, such as $L i([3],[20])$ (researchers prefer to study crystals, where the isotope effects are large and it is easier to carry out appropriate measurements) show that there is square-root dependence of the force constants on the isotope mass, which was required by Eq.(79). The same dependence of the force constants on the mass of the isotope has been found in tin [21].

Unfortunately, no direct experiments of the effect of isotopic substitution on the electronic properties (such as the electronic specific heat and the Fermi energy), on metals of interest to us have so far been conducted.

Let us consider what should be expected in such measurements. A convenient choice for the superconductor is mercury, as it has many isotopes and their isotope effect has been carefully measured back in the 50s of the last century as aforementioned.

The linear dependence of the critical temperature of a superconductor on its Fermi energy (Eq.(20)) and also the existence of the isotope effect suggests the dependence of the ion density in the crystal lattice from the mass of the isotope. Let us consider what should be expected in such measurements.

Even then, it was found that the isotope effect is described by Eq.(79) in only a few superconductors. In others, it displays different values, and therefore in a general case it can be described by introducing of the parameter $\mathfrak{a}$ :

$$
M_{i}^{\mathfrak{a}} T_{c}=\text { Const. }
$$

At taking into account Eq.(20), we can write

$$
T_{c} \sim \mathcal{E}_{F} \sim n_{e}^{2 / 3} .
$$

The parameter $l$ which characterizes the ion lattice obtains an increment $\Delta l$ with an isotope substitution:

$$
\frac{\Delta l}{l}=-\frac{\mathfrak{a}}{2} \cdot \frac{\Delta M_{i}}{M_{i}}
$$

where $M_{i}$ and $\Delta M_{i}$ are the mass of isotope and its increment.

It is generally accepted that in an accordance with the terms of the phonon mechanism, the parameter $\mathfrak{a} \approx \frac{1}{2}$ for mercury. However, the analysis of experimental data [1]-[2] (see Fig.(Part I-4)) shows that this parameter is actually closer to $1 / 3$. Accordingly, one can expect that the ratio of the mercury parameters is close to:

$$
\frac{\left(\frac{\Delta l}{l}\right)}{\left(\frac{\Delta M_{i}}{M_{i}}\right)} \approx-\frac{1}{6}
$$

\section{REFERENCES}

[1] Maxwell E. : Phys.Rev.,78,p 477(1950).

[2] Serin et al : Phys.Rev.B,78,p 813(1950).

[3] Inyushkin A.V. : Chapter 12 in "Isotops" (Editor Baranov V.Yu), PhysMathLit, 2005 (In Russian).

[4] Vasiliev B.V. : Superconductivity as a consequence of an ordering of the electron gas zero-point oscillations, Physica C, 471,277-284 (2011)

[5] Vasiliev B.V. : Superconductivity and condensation of ordered zero-point oscillations, Physica C, 471,277-284 (2012)

[6] Vasiliev B.V. : "Superconductivity, Superfluidity and Zero-Point Oscillations" in "Recent Advances in Superconductivity Research" , pp.249-280, Nova Publisher,NY(2013)

[7] Bardeen J.: Phys.Rev.,79,p. 167-168(1950).

[8] Shablo A.A. et al: Letters JETPh, v.19, 7,p.457461 (1974)

[9] Sharvin D.Iu. and Sharvin Iu.V.: Letters JETPh, v.34, 5, p.285-288 (1981)

[10] Vasiliev B.V. and Luboshits V.L.: Physics-Uspekhi, 37, 345, (1994)

[11] Pool Ch.P.Jr : Handbook of Superconductivity, Academic Press, (2000)

[12] Ketterson J.B. and Song S.N.: Superconductivity, Cambridge (1999) 
[13] Landau L.D. and Lifshits E.M.: Statistical Physics, 1, 3rd edition, Oxford:Pergamon, (1980)

[14] Kittel Ch. : Introduction to Solid State Physics, Wiley (2005)

[15] Abragam-Becker : Teorie der Elektizität, Band 1, Leupzig-Berlin, (1932)

[16] Albert Messiah: Quantum Mechanics (Vol. II), North Holland, John Wiley and Sons. (1966)

[17] Linton E.A. : Superconductivity, London: Mathuen and Co.LTDA, NY: John Wiley and Sons Inc., (1964)
[18] Golovashkin A.I. : Preprint PhIAN, 10, Moscou, 2005 (in Russiian).

[19] Ashcroft N.W., Mermin N.D.: Solid state physics, v 2., Holt,Rinehart and Winston, (1976)

[20] Kogan V.S.: Physics-Uspekhi, 78579 (1962)

[21] Wang D.T. et al : Phys.Rev.B,56,N 20,p. 13167(1997) 